\title{
Genetic and Environmental Influences on Self-Esteem in a Japanese Twin Sample
}

\author{
Toshimitsu Kamakura', Juko Ando² and Yutaka Ono ${ }^{3}$ \\ ' Faculty of Letters, Chiba University, Chiba, Japan \\ ${ }^{2}$ Faculty of Letters, Keio University, Tokyo, Japan \\ ${ }^{3}$ School of Medicine, Keio University, Tokyo, Japan
}

\begin{abstract}
The purpose of the present study is to clarify the mechanism of Japanese self-esteem (SE) in genetic and environmental influences using twin methodology. Eighty-one pairs of adolescent twins, including 50 pairs of monozygotic (MZ) twins and 31 pairs of dizygotic (DZ) twins, participated in this study. Self-esteem was assessed using the Rosenberg Self-Esteem Scale (RSES), translated into Japanese. As a result of using univariate twin analyses, model comparisons using the Akaike Information Criterion (AIC) indicated that the $A E$ model was the best fit $(A I C=-5.35)$. In the best-fitting $A E$ model, the heritability $\left(a^{2}\right)$ of SE was revealed to be moderate, accounting for $49 \%$ of the variance; environmental influences (individual-specific environmental factors) explained $51 \%$ of the variance. These results are consistent with the findings of some behavioral genetics studies of SE in the West and show that there is no difference between Western and Japanese populations in the mechanism of SE considering genetic and environmental influences. The results also suggest the importance of considering both genetic and environmental factors in studies of Japanese SE.
\end{abstract}

Self-esteem (SE) in the psychological field is taken as a part of the self-concept and a feeling of self-evaluation, and is a person's positive or negative attitude toward him/ herself (Rosenberg, 1965). Numerous studies of SE have been performed across many fields such as clinical and social psychology. Some previous studies in Europe and America, for instance, showed that high levels of SE have a negative relationship with delinquency (Rosenberg \& Rosenberg, 1978), anxiety disorders (Ingham et al., 1986), eating disorders (Kendler et al., 1991), and major depression (Brown et al., 1986). Some previous studies in Japan also showed that high levels of SE have a negative relationship with loneliness (Kudou \& Nisikawa, 1983), anthrophobic-tendency (Okada \& Nagai, 1989), conformity (Kurosawa, 1993) and shyness (Aikawa, 1991). However, there have been few empirical attempts to investigate the mechanism of SE.

Robson (1988) regarded self-concept as formed through an interaction with other people rather than inborn. He also suggested that race would influence the mechanism of SE. In this way, the self has been examined from the cultural perspective, including an interaction with others. It is presumed that the self is viewed differently in Western and non-Western cultures (Kitayama, 1994). In Western cultures, the independent view of the self is gener- ally that an individual is separate from others and insists on uniqueness. On the other hand, in non-Western cultures, the interdependent view of the self is predominantly that individuals are connected with each other, are less differentiated from others, and become part of various interpersonal relationships. Markus and Kitayama (1991) also argued that a cultural difference in the view of the self was related to the basis of SE.

Contrary to this perspective, behavioral genetic studies have focused on examining the genetic and environmental influences on self-concept and ego development (Hur et al., 1998; Newman et al., 1998). Some behavioral genetic studies in the West showed that genetic factors moderately contribute to the mechanism of SE. Roy et al. (1995) computed genetic and environmental determinants of SE in the population, based on the sample from the Virginia Twin Register of Caucasian, using Rosenberg's Self-Esteem Scale (RSES; Rosenberg, 1965). Two longitudinal studies showed that the contributions of genetic and environmental (E; individual-specific environmental) factors to $\mathrm{SE}$ were each about 50\%. Kendler et al. (1998) also examined, using the RSES measure and the twin method, the contribution of genetic and environmental factors to SE separately in men and women. The results indicated that: (1) individual differences in SE of both men and women were best explained by genetic and individual-specific environmental factors; (2) the genetic contribution to the variance of men (29\%) was similar to that of women (32\%).

Thus, behavioral genetic studies suggest that genetic factors contribute to the mechanism of Western SE moderately. In contrast, Markus and Kitayama (1991) strongly emphasized that environmental factors influence the basis of Japanese SE; that is, the mechanism of Japanese SE may be formed by environmental factors for the most part and only slightly affected by genetic factors. However, if the mechanism of SE considering genetic and environmental factors in Japanese population is not different from that in Western population, the mechanism of Japanese SE, as well as Western SE, can be influenced moderately by genetic factors.

Address for correspondence: T. Kamakura, 5-18-9 Tamagawa, Chofu, Tokyo 182-0025, Japan.E-mail: k.kamakura@nifty.com 
The purpose of the present study is to examine the mechanism of Japanese SE in genetic and environmental influences using a Japanese twin sample representing nonWestern populations. Using the twin method, we can show whether the mechanism of SE in genetic and environmental influences is different between Western and Japanese populations. In order to estimate the contributions of genetic and environmental factors to the observed individual differences in Japanese SE, we applied behavioral genetic analyses to the corresponding covariance data.

\section{Method}

Sample

The data reported here were obtained from the twins who participated in the Keio Twin Project (KTP), a comprehensive research project in human behavioral genetics that focused on cognitive abilities and molecular genetics as well as personality using a large Japanese sample of twins (Ando \& Ono, 1998). The KTP has made a residential information list. To date, data describing 5200 pairs of twins who live in and near Tokyo have already been obtained. These twin samples living in and near Tokyo represent the whole sample of Japanese twins, since people who come from various areas in Japan are concentrated in Tokyo. This list is named the Keio Twin Registry (KTR), and contains the names, addresses and birth dates of twins who were born on the same day and live together, ranging from newborns to those over forty years of age. These data were obtained from official residential records, which are available at city halls. We assume that, as the factor of twins living together is constant, the amount of contact is not influencing twin similarity in SE.

Invitation letters to participate in our project were sent to approximately 2000 pairs of twins in the KTR ranging in age from 14 to 28 years old. The letter informed them that in this project they would be ask to complete a onehour questionnaire at home and undergo a three-hour assessment of cognitive ability and personality inventory including taking blood samples at the University.

The present research is based on the second of the two waves of the research. In Wave I, over 300 pairs agreed to participate. After all the subjects received written explanations of the purpose of the research, the research items, protection of their privacy and their right to cancel their participation at any time if they wished, they filled in the written informed consent document. Subjects under 20 years of age were required to obtain their parents' written consent as well. This constituted the largest twin study conducted in Japan in the past three decades. In Wave II, 81 pairs of twins participated. Of the 81 pairs, 50 pairs were monozygotic (MZ) twins, 24 pairs were same-sex dizygotic (DZ) twins, and 7 pairs were opposite-sex DZ twins. Zygosity was determined by twins' physical resemblance (Ooki et al., 1991) and by polymorphism of D4DR and serotonin transporter genes, which are verified to be of over $99 \%$ accuracy as a determinant.

\section{Measurement}

Self-esteem was assessed by the 10-item Rosenberg SelfEsteem Scale (RSES; Rosenberg, 1965) translated into
Japanese (Yamamoto et al., 1982). Subjects were asked to "indicate the extent to which you think each item describes yourself". This measurement used a five-point Likert scale from strongly agree (5) to strongly disagree (1).

\section{Analytical Procedure}

The effect of additive genetic factors (A) is assumed to be the sum of multiple genes (polygene) whose effects are small and additive to form a quantitative phenotype. Because MZ twins share all their genes and DZ twins share on average only half of their genes, additive genetic effects cause intraclass correlation in MZ twins to be twice as large as that in DZ twins. The effect of nonadditive genetic factors (D) is assumed to be an interactive (nonadditive) contribution of alleles within a single locus (dominance). Shared environment (C) is a variable that makes family members alike in the common environment shared by all family members. Because we are assuming that $M Z$ twins and DZ twins share their environment to the same extent, (C) causes the correlation in MZ twins and $\mathrm{DZ}$ twins to be equal. Individual specific environment ( $E$ or nonshared environment) is a variable that makes family members different even if they live together. It also includes measurement errors.

Using goodness-of-fit statistics, we specify a genetic model to be tested (e.g., ACE, ADE, AE, CE). The ACE model in phenotypic covariance is explained by additive genetic $(A)$, shared environment $(C)$, and individual specific environment $(\mathrm{E})$; the $\mathrm{ADE}$ model is explained by $\mathrm{A}, \mathrm{D}$ and $\mathrm{E}$; the $\mathrm{AE}$ model is explained by $\mathrm{A}$ and $\mathrm{E}$; the $\mathrm{CE}$ model is explained by $\mathrm{C}$ and $\mathrm{E}$. We fit the $\mathrm{ACE}, \mathrm{ADE}, \mathrm{AE}$ and $\mathrm{CE}$ model. The choice of the preferred model is based on Akaike's information criteria (AIC) computed as $\chi^{2}-2 d f$ (where $d f$ represents the degrees of freedom). The AIC parameter is calculated for each model, and reflects a model's goodness of fit as well as its parsimony. The model that results in the smallest AIC is regarded as the best fit.

\section{$\overline{\text { Result }}$}

\section{Factor Analysis}

After factor analysis of the 10-item RSES, 9 items remained, with an alpha coefficient of 0.87 . The loadings from the principal factor obtained from the principal component analysis are shown in Table 1 . This one factor explained $51 \%$ of the variance of the 9 items.

\section{Descriptive Statistics}

The sum of the scores of 9 items remaining after the factor analysis was used as the RSES score. It ranged from 9 to 45. Table 2 shows the means, standard deviations, maximum and minimum of the RSES score in the twin sample. Twin intraclass correlation coefficients for SE were 0.48 in MZ twins and 0.08 in DZ twins. Also, the standard errors for the twin pair correlations with SE were 0.11 in MZ twins and 0.15 in DZ twins. Using $t$ tests, we found no significant sex differences on the mean of RSES score between males and females in the twin sample. 
Table 1

Factor Loading of Rosenberg Self-Esteem Scale

\begin{tabular}{lr}
\hline Item & $\begin{array}{c}\text { Factor } \\
\text { Loading }\end{array}$ \\
\hline
\end{tabular}

I feel that I am a person of worth, at least equal with others

I feel I have a number of good qualities

0.77

All in all, I am inclined to think that I am a failure

$-0.62$

I am able to do things as well as most other people

0.68

I feel that I do not have much to be proud of

$-0.70$

I take a positive attitude toward myself

0.62

On the whole, I am satisfied with myself

0.69

At times, I think I am no good at all

$-0.72$

I certainly feel useless at times

$-0.81$

Table 2

Statistics for Rosenberg's Self-Esteem Scale (RSES) by Zygosity

\begin{tabular}{lccc}
\hline & $\begin{array}{c}\text { Total } \\
(N=81 \text { pairs })\end{array}$ & $\begin{array}{c}\mathrm{MZ} \\
(N=50 \text { pairs })\end{array}$ & $\begin{array}{c}\mathrm{DZ} \\
(N=31 \text { pairs })\end{array}$ \\
\hline Means & 30.13 & 30.34 & 29.79 \\
Standard deviations & 6.44 & 6.16 & 6.92 \\
Maximum & 45 & 45 & 44 \\
Minimum & 11 & 15 & 11 \\
\hline
\end{tabular}

\section{Univariate Model-Fitting}

To understand how genetic and environmental factors contribute to SE, we fitted a number of univariate twin models, as shown in Table 3. We eliminated the data on opposite-sex DZ twins in the analyses. Here we systematically compare the $\mathrm{ACE}, \mathrm{ADE}, \mathrm{AE}$ and $\mathrm{CE}$ models for the RSES score.

For the RSES score, the ADE model fitted well $\left(\chi^{2}=\right.$ 2.58 , AIC $=-3.42)$. The AE model had an identical goodness-of-fit $\chi^{2}$ to that of the ACE model. However, the AIC indicated that the AE model was the best fit (AIC = -5.35). Also, the $\chi^{2}$ parameter indicated that the AE model was the most parsimonious, as the fit did not significantly worsen when $\mathrm{D}$ was dropped from the model. The CE model fitted the data worse than the ACE model $\left(\chi^{2}=4.78\right.$, AIC $=-3.22)$. For the best-fitting AE model, the heritability $\left(\mathrm{a}^{2}\right)$

\section{Table 3}

Univariate Twin Models of Self-esteem (Same-sex Twins)

\begin{tabular}{lcccccc}
\hline Model & $\chi^{2}$ & $d f$ & AIC & $a^{2}$ & $c^{2}$ & $\mathrm{e}^{2}$ \\
\hline ACE & 2.65 & 3 & -3.35 & & & \\
ADE & 2.58 & 3 & -3.42 & & & \\
AE & 2.65 & 4 & -5.35 & 0.49 & - & 0.51 \\
CE & 4.78 & 4 & -3.22 & & & \\
\hline
\end{tabular}

of SE explained $49 \%$ of the variance and individual specific environmental effects $\left(\mathrm{e}^{2}\right)$ explained $51 \%$ of the variance.

\section{Discussion}

The present study examined the mechanism of Japanese self-esteem by using behavioral genetic analyses. The results of this study showed that genetic factors contribute to individual differences in Japanese SE moderately. Our results were consistent with the findings of behavioral genetic studies of SE in the West; Japanese SE was moderately heritable $(49 \%)$ and was affected by individual specific environment $(51 \%)$. These findings also indicate that there is no difference in the mechanism of SE in genetic and environmental influences between Western and Japanese population.

It seems that the mechanism of SE is not dependent on cultural background but is similar to personality traits or attitudes. For instance, some behavioral genetic studies have shown that in personality traits heritability accounts for $40 \%$ to $50 \%$ of variance and individual specific environmental (non-shared environment) factors explains 40\% to $50 \%$ of variance (Harris, 1995; Plomin et al., 1994). As the contributions of the two factors to SE obtained in this study were similar to these findings on personality traits, SE may be regarded as being a personality trait or attitude.

Although the results of this study suggest that genetic factors have a moderate influence on Japanese SE, little is known about genetic effects of SE. One possible explanation for genetic effects on SE is that genetic factors contribute to it directly. However, genes that have a direct relationship to SE have not been found. Another possible explanation is that genetic factors indirectly contribute to SE. For example, it is possible that SE has a relationship with personality or psychological traits that have been reported to be heritable in previous studies. Roberts and Kendler (1999) found by trivariate twin modeling that the covariation of SE, neuroticism and major depression is due largely to genetic factors. However, they also reported that neuroticism is a stronger predictor of genetic factors for major depression than SE. Therefore, to find indirectly genetic effects of SE, we need to examine the relationship between $\mathrm{SE}$ and heritable traits by applying multivariate genetic models.

The results of this study also indicate the importance of individual specific environmental influences on Japanese SE. This means that it is possible to change Japanese SE in any case. For example, if an intervention works on individual specific environmental factors successfully, it might help to increase low levels of SE.

There are some methodological limitations to the present study. Firstly, although we used RSES to measure Japanese SE, we need to examine Japanese SE further by using another measurement. Second, in the present study, we examined the mechanism of Japanese SE in genetic and environmental influences using Japanese twin sample as representing non-Western twin samples. However, there are multiple cultural view of the self among non-Western cultures (Takada, 1999), so the mechanism of SE in genetic and environmental influences might vary among nonWestern populations. It is necessary, therefore, to 
investigate the mechanism of SE in genetic and environmental influences using other non-Western populations, for instance, Korean or Chinese. Third, this study has a small number of subjects. It seems that the response rate of the samples is not high, due to a typical property of the Japanese people.

In conclusion, the findings of the present study, consistent with the results of some behavioral genetics studies in the West suggesting the effects of genetic and environmental factors moderately contribute to SE, indicate that the mechanism of SE in Japanese population considering genetic and environmental influences is not different from that in Western population. These findings also suggest the importance of both genetic and environmental factors that contribute to Japanese SE.

\section{References}

Aikawa, A. (1991). A study on reliability and validity of a scale to measure shyness as a trait. Japanese Journal of Psychology, 62, $149-155$.

Ando, J., \& Ono, Y. (1998). Keio Twin Project: A preliminary report (Abstract of the 9th International Congress on Twin Studies). Twin Research, 1(1), 81.

Brown, G. W., Andrews, B., Harris, T., Adler, Z., \& Bridge, L. (1986). Social support, self-esteem and depression. Psychological Medicine, 16, 813-831.

Harris, J. R. (1995). Where is the child's environment? A group socialization theory of development. Psychological Review, 102, 458-489.

Hur, Y. M., McGue, M., \& Iacono, W. G. (1998). The structure of self-concept in female preadolescent twins: A behavioral genetics approach. Journal of Personality and Social Psychology, 74, 1069-1077.

Ingham, J. G., Kreitman, N. B., Miller, P. M., Saahidharan, S. P., \& Surtees, P. G. (1986). Self-esteem, vulnerability and psychiatric disorder in the community. British Journal of Psychiatry, 148, 375-385.

Kendler, K. S., Gardner, C. O., \& Prescott, C. A. (1998). A population-based twin study of self-esteem and gender. Psychological Medicine, 28, 1403-1409.

Kendler, K. S., MacLean, C. J., Neale, M. C., Kessler, R. C., Heath, A. C., \& Eaves, L. J. (1991). The genetic epidemiology of bulimia nervosa. American Journal of Psychiatry, 148, $1627-1637$.
Kitayama, S. (1994). Cultural views of self and psychological processes. Japanese Journal of Social Psychology, 10, 153-167.

Kudou, T., \& Nisikawa, M. (1983). A study of the feeling of loneliness (I). The reliability and validity of the revised UCLA loneliness scale. Japanese Journal of Experimental Social Psychology, 22, 99-108.

Kurosawa, K. (1993). The effect of self-consciousness and selfesteem on conformity to a majority. Japanese Journal of Psychology, 63, 379-387.

Markus, H. R., \& Kitayama, S. (1991). Culture and the self: Implications for cognition, emotion, and motivation. Psychological Review, 98, 224-253.

Newman, D. L., Tellegen, A., \& Bouchard, Jr., T. J. (1998). Individual difference in adult ego development: Sources of influence in twin reared apart. Journal of Personality and Social Psychology, 74, 985-1095.

Okada, T., \& Nagai, T. (1989). Self-esteem and anthrophobic-tendency in adolescents. Japanese Journal of Psychology, 60, 386-389.

Ooki, S., Yamada, K., \& Asaka, A. (1991). Zygosity diagnosis of twins by questionnaire for twin's mothers. Shoni Hoken Keikyu, 50, 71-76

Plomin, R., Owen, M. J., \& McGuffin, P. (1994). The genetic basis of complex human behaviors. Science, 264, 1733-1739.

Robert, S. B., \& Kendler, K. S. (1999). Neuroticism and selfesteem as indices of the vulnerability to major depression in woman. Psychological Medicine, 29, 1101-1109.

Robson, P.J. (1988). Self-esteem - a psychiatric view. British Journal of Psychiatry, 153, 6-15.

Rosenberg, F. R., \& Rosenberg, M. (1978). Self-esteem and delinquency. Journal of Youth and Adolecence, 7, 279-294.

Rosenberg, M. (1965). Society and the adolescent self-image. Princeton, NJ: Princeton University Press.

Roy, M. A., Neale, M. C., \& Kendler, K. S. (1995). The genetic epidemiology of self-esteem. British Journal of Psychiatry, 166, 813-820.

Takada, T. (1999). Developmental process of independent and interdependent self-construal in Japanese culture: Cross-cultural and cross-sectional analyses. Japanese Journal of Educational Psychology, 47, 480-489.

Yamamoto, M., Matui, Y., \& Yamanari, Y. (1982). The structure of perceived aspects of self. Japanese Journal of Educational Psychology, 30, 64-68. 